\title{
Monomicrobial Non-Neutrocytic Bacteriascites due to Salmonella enteritidis: A Case Report and Literature Review
}

\author{
Samran Haider ${ }^{\mathrm{a}, \mathrm{c}}$, Rohit Gupta ${ }^{\mathrm{a}}$, Aditya Sood ${ }^{\mathrm{a}}$, Amaraja Kanitkar ${ }^{\mathrm{a}}$, \\ Ghulam Saydain $^{\mathrm{b}}$
}

\begin{abstract}
Spontaneous bacterial peritonitis (SBP) is diagnosed on the basis of neutrophilic leukocytosis in peritoneal fluid. In monomicrobial nonneutrocytic bacteriascites (MNB), bacterial cultures are positive in absence of neutrophilic leukocytosis (polymorphonuclear neutrophil $\left.(\mathrm{PMN})<250 / \mathrm{mm}^{3}\right)$. Salmonella is an uncommon cause of SBP and only one other case of Salmonella MNB has been reported. We report such a case of Salmonella MNB. A 70-year-old man with diabetes mellitus and cirrhosis was brought to the ED with irritability and confusion for 2 days. He had recovered from a diarrheal illness 3 days prior to presentation. Examination was notable for tachycardia, tachypnea, confusion, and ascites with diffuse abdominal tenderness. Pertinent investigations included creatinine $1.38 \mathrm{mg} / \mathrm{dL}$, ammonia $142 \mathrm{mMol} / \mathrm{L}$, and lactic acid $5.3 \mathrm{mMol} / \mathrm{L}$. Diagnostic paracentesis revealed a nucleated cell count of $33 / \mathrm{mm}^{3}$. He was admitted to MICU for hepatic encephalopathy and severe sepsis and started on empiric vancomycin and cefepime. Salmonella enteritidis was isolated in the ascitic fluid culture on day 4. Repeat paracentesis was indicated but could not be performed because he was transferred to another hospital. Infection secondary to non-typhoidal Salmonella usually presents as a self-limited gastrointestinal disease. The diagnosis of MNB is made when ascitic fluid culture is positive but fluid PMN count is < $250 / \mathrm{mm}^{3}$. Thirty-eight percent of patients with MNB eventually progress to SBP, while in the rest, this represents spontaneous colonization resolving without antibiotic treatment. Mechanism of infection is translocation of bacteria across intestinal cells into the peritoneum, increasing according to the Child-Pugh classification. Treatment is recommended if patients continue to have positive ascitic fluid cultures on subsequent paracentesis or if they develop local or systemic signs of infection. MNB is a potentially life-threatening condition with mortality similar to SBP. Clinicians must follow ascitic fluid culture even when fluid PMN count is $<250 / \mathrm{mm}^{3}$.
\end{abstract}

Manuscript accepted for publication June 30, 2016

aDetroit Medical Center/Wayne State University, University Health Center, 4201 Saint Antoine Street, Room 2E, Detroit, MI 48201, USA

${ }^{b}$ Detroit Receiving Hospital, Pulmonary Hypertension Program at Detroit Medical Centre/Wayne State University, Detroit, MI 48201, USA

${ }^{\mathrm{c} C}$ Corresponding Author: Samran Haider, Detroit Medical Center/Wayne State University, University Health Center, 4201 Saint Antoine Street, Room 2E, Detroit, MI 48201, USA. Email: shaider@med.wayne.edu

doi: http://dx.doi.org/10.14740/jmc2575w
Keywords: Spontaneous bacterial peritonitis; Cirrhosis; Ascites; Neutrascites; Monomicrobial non-neutrocytic bacteriascites; Salmonella

\section{Introduction}

Spontaneous bacterial peritonitis (SBP) is diagnosed on the basis of neutrophilic leukocytosis in peritoneal fluid. Antimicrobial therapy is initiated based on this finding pending isolation of organism. In some cases, bacteria may exist in peritoneal fluid in absence of neutrophilic leukocytosis (polymorphonuclear neutrophil $(\mathrm{PMN})<250 / \mathrm{mm}^{3}$ ), a condition termed as monomicrobial non-neutrocytic bacteriascites (MNB). This is a poorly recognized clinical entity. Salmonella has been reported as an uncommon cause of SBP and is a rare microorganism to cause MNB. We report a rare case of Salmonella MNB and present a brief review of literature.

\section{Case Report}

A 70-year-old man was brought to the hospital by his son with irritable mood and confusion for 2 days. The patient had recently recovered from a non-bloody diarrheal illness 3 days prior to presentation for which he did not receive any medical care. His past medical history was significant for non-insulin dependent diabetes mellitus, and Child-Pugh class $\mathrm{C}$ cirrhosis secondary to autoimmune hepatitis diagnosed 7 years ago for which he did not receive any treatment. Physical examination revealed blood pressure of $142 / 88$, heart rate of 135 beats per minute, respiratory rate of 26 breaths per minute and dry oral mucosa. Patient was sleepy but arousable with marked confusion, incoherent speech and inappropriate responses to questions. Chest auscultation showed regular heart beat with tachycardia, normal heart and breath sounds. Abdominal examination was notable for distension, non-tense ascites with diffuse tenderness on palpation. Bowel sounds were auscultated in all four quadrants. Initial laboratory tests were significant for hemoglobin $10.2 \mathrm{~g} / \mathrm{L}$, white blood cell count $6,800 / \mathrm{mm}^{3}$ ( $72 \%$ neutrophils), platelets $130,000 / \mathrm{mm}^{3}$, sodium $133 \mathrm{mg} /$ $\mathrm{dL}$, creatinine $1.38 \mathrm{mg} / \mathrm{dL}$, ammonia $142 \mathrm{mMol} / \mathrm{L}$, lactic acid $5.3 \mathrm{mMol} / \mathrm{L}$, ALT 39 units/L, AST 67 units/L, ALP 82 units/L, total bilirubin $1.9 \mathrm{mg} / \mathrm{dL}$, direct bilirubin $0.8 \mathrm{mg} / \mathrm{dL}$, total pro- 
Table 1. Causative Microorganisms of Spontaneous Bacterial Peritonitis and Monomicrobial Non-Neutrocytic Bacteriascites [14]

\begin{tabular}{lll}
\hline Microorganism & SBP (\%) & Bacteriascites (\%) \\
\hline Escherichia coli & 37 & 27 \\
Klebsiella pneumoniae & 17 & 11 \\
Pneumococcus & 12 & 9 \\
Streptococcus viridans & 9 & 2 \\
Staphylococcus aureus & 0 & 7 \\
Miscellaneous gram negative & 10 & 14 \\
\hline
\end{tabular}

tein $6.0 \mathrm{~g} / \mathrm{dL}$, and albumin $2.1 \mathrm{~g} / \mathrm{dL}$. Urinalysis showed 3+ leukocyte esterase, 20 - 50 white blood cells per high power field, and positive urine nitrite. Blood and urine cultures were negative. Diagnostic paracentesis was performed which revealed a nucleated cell count of $33 / \mathrm{mm}^{3}$ (13\% neutrophils) and proteins $<2 \mathrm{~g} / \mathrm{dL}$. The initial clinical impression was severe sepsis; patient was started on empiric vancomycin and cefepime. Salmonella enteritidis was isolated in the ascitic culture on day 4. Therapy with cefepime was thought to cover this organism. Patient was treated with lactulose and rifaximin for hepatic encephalopathy but had a poor response. His clinical condition worsened and he subsequently developed septic shock secondary to pneumonia caused by ESBL producing E. coli. He required vasopressors, mechanical ventilation and renal replacement therapy. Antibiotics coverage was adjusted according to susceptibilities. On day 5, he was transferred to another facility for continued care per family's request where he died 6 days later. Repeat paracentesis was indicated but could not be performed as the patient was transferred.

\section{Discussion}

Infection secondary to non-typhoidal Salmonella is uncommon in the United States and usually presents as a self-limited gastrointestinal disease. SBP secondary to Salmonella is uncommon. It has been reported previously in patients with immunosuppression and may present without signs or symptoms of SBP [1-4]. We present a rare case of MNB with isolation of Salmonella enteritidis from the ascitic fluid. To our knowledge, this is only the second case report for MNB secondary to Salmonella enteritidis [5].

The diagnosis of MNB is made when ascitic fluid culture is positive but $\mathrm{PMN}$ count is $<250 / \mathrm{mm}^{3}$. MNB is a poorly recognized clinical entity. Small studies show prevalence of asymptomatic MNB between $0.7 \%$ and $3 \%$ in patients with compensated hepatic cirrhosis [6-8], while prevalence among patients with decompensated hepatic cirrhosis is reported to be as high as $16.25 \%$ [9]. Mechanism of infection has been widely cited as translocation of bacteria across intestinal cells [10, 11]. The prevalence of bacterial translocation increases according to the Child-Pugh classification, up to $30.8 \%$ in Child $\mathrm{C}$ patients [12]. The opsonic activity in ascitic fluid depends on the protein content. Low-protein ascitic fluids in cirrhotic patients are deficient in opsonic activity and are particularly predisposed to ascitic fluid infections [13]. Total protein in the ascitic fluid in our patient was reported as $<2 \mathrm{~g} / \mathrm{dL}$. The fact that he had a diarrheal illness preceding presentation might have led to translocation of Salmonella into the peritoneum. In general, gram negative bacteria are the primary causative microorganisms for MNB, similar to SBP (Table 1) [14].

The clinical course of MNB is variable. Runyon et al report that $38 \%$ of patients with MNB eventually progress to SBP [14] and hence, a second diagnostic paracentesis is recommended after $72 \mathrm{~h}$. In other patients, it represents spontaneous colonization of ascites which resolves without antibiotic treatment. Treatment with antibiotics is recommended if patients continue to have positive ascitic fluid cultures on subsequent paracentesis or if they develop local or systemic signs of infection [15]. Mortality rate for MNB is also reported to be similar to SBP [16].

\section{Conclusion}

MNB is a poorly recognized clinical entity with variable course. Clinicians must timely recognize MNB as mortality is similar to SBP. To avoid missing MNB, ascitic fluid cultures must be sent and vigilantly followed up even with nucleated cell count of $<250 / \mathrm{mm}^{3}$ in the ascitic fluid. MNB may progress to SBP and hence, a repeat paracentesis should be done after 72 h. Salmonella is an uncommon cause of SBP and rarely causes MNB but should be considered in immunocompromised hosts.

\section{Conflicts of Interest}

None.

\section{References}

1. Adhikary R, Joshi S, Venugopa RV, Ganjoo N. Spontaneous bacterial peritonitis caused by S. paratyphi A. J Assoc Physicians India. 2013;61(12):930-931.

2. Rajekar H, Wai CT, Lee KH, Wong SY, Tan KC. Spontaneous bacterial peritonitis from Salmonella: an unusual bacterium with unusual presentation. Hepatol Int. 2008;2(3):388-389.

3. de la Fuente-Aguado J, Bordon J, Esteban AR, Aguilar A, Moreno JA. Spontaneous non-typhoidal Salmonella peritonitis in patients with serious underlying disorders. Infection. 1999;27(3):224-227.

4. Joseph T, Sobhan P, Bahuleyan S, John A, Abdul Sathar S, Sreesh S, Vinayakumar KR. Non-typhoidal salmonella: an unusual cause of spontaneous bacterial peritonitis in decompensated cirrhosis. Gastroenterol Rep (Oxf). 2014;2(3):242-244.

5. Lopez-Arce G, Torre-Delgadillo A, Tellez-Avila FI. Salmonella sp group A: a rare cause of bacterascites. A case report. Ann Hepatol. 2008;7(3):260-261.

6. Mohan P, Venkataraman J. Prevalence and risk factors for 
unsuspected spontaneous ascitic fluid infection in cirrhotics undergoing therapeutic paracentesis in an outpatient clinic. Indian J Gastroenterol. 2011;30(5):221-224.

7. Evans LT, Kim WR, Poterucha JJ, Kamath PS. Spontaneous bacterial peritonitis in asymptomatic outpatients with cirrhotic ascites. Hepatology. 2003;37(4):897-901.

8. Castellote J, Girbau A, Maisterra S, Charhi N, Ballester $\mathrm{R}$, Xiol X. Spontaneous bacterial peritonitis and bacterascites prevalence in asymptomatic cirrhotic outpatients undergoing large-volume paracentesis. J Gastroenterol Hepatol. 2008;23(2):256-259.

9. Kasztelan-Szczerbinska B, Slomka M, Celinski K, Serwacki M, Szczerbinski M, Cichoz-Lach H. Prevalence of spontaneous bacterial peritonitis in asymptomatic inpatients with decompensated liver cirrhosis - a pilot study. Adv Med Sci. 2011;56(1):13-17.

10. Runyon BA, Squier S, Borzio M. Translocation of gut bacteria in rats with cirrhosis to mesenteric lymph nodes partially explains the pathogenesis of spontaneous bacte- rial peritonitis. J Hepatol. 1994;21(5):792-796.

11. Finlay BB, Falkow S. Virulence factors associated with Salmonella species. Microbiol Sci. 1988;5(11):324-328.

12. Cirera I, Bauer TM, Navasa M, Vila J, Grande L, Taura P, Fuster J, et al. Bacterial translocation of enteric organisms in patients with cirrhosis. J Hepatol. 2001;34(1):32-37.

13. Runyon BA, Morrissey RL, Hoefs JC, Wyle FA. Opsonic activity of human ascitic fluid: a potentially important protective mechanism against spontaneous bacterial peritonitis. Hepatology. 1985;5(4):634-637.

14. Koulaouzidis A, Bhat S, Karagiannidis A, Tan WC, Linaker BD. Spontaneous bacterial peritonitis. Postgrad Med J. 2007;83(980):379-383.

15. Runyon BA. Monomicrobial nonneutrocytic bacterascites: a variant of spontaneous bacterial peritonitis. Hepatology. 1990;12(4 Pt 1):710-715.

16. Chu CM, Chang KY, Liaw YF. Prevalence and prognostic significance of bacterascites in cirrhosis with ascites. Dig Dis Sci. 1995;40(3):561-565. 